\title{
MRI findings at image guided adaptive cervix cancer brachytherapy: radiation oncologist's perspective
}

\author{
Primoz Petric, MD, Noora Mohammed-Al-Hammadi, MD \\ Department of Radiation Oncology, National Center for Cancer Care and Research, Doha, Qatar
}

\begin{abstract}
Magnetic resonance imaging (MRI) represents the reference imaging modality for image guided adaptive brachytherapy (IGABT) of cervix cancer. Accurate interpretation of pre-treatment MRI is required for proper understanding of the tumor extent and topography at IGABT. Planning and optimal timing of the application begins already before treatment, and may need to be adapted during external beam irradiation (EBRT) according to additional clinical and/or radiological findings. The level of MRI utilization in IGABT depends on the infrastructural capabilities of individual centers, ranging from no use at all to repetitive imaging during EBRT and each IGABT fraction. In this article, we summarize the role of different imaging modalities and practical aspects of MRI interpretation in cervix cancer IGABT, concentrating on the systematic evaluation of post-insertion images. MRI with the applicator in place from the radiation oncologist's perspective should begin with immediate identification of eventual complications of the application procedure and assessment of the implant adequacy, followed by appropriate corrective measures in case of adverse findings. Finally, the tumor extent, topography, and treatment response should be evaluated in the context of initial clinical and radiological findings to allow for an appropriate selection and delineation of the target volumes.

Key words: brachytherapy, cervix cancer, magnetic resonance imaging.

\section{Purpose}

The conventional approach to cervix cancer brachytherapy (BT) is based on acquisition of two orthogonal pelvic radiographs after the intracavitary applicator insertion. The capability for treatment plan optimization with this technique is limited. The optimization process typically aims at achieving an adequate dose at the Manchester point A while keeping the doses at the bladder and rectum ICRU (International Commission on Radiology Units) points below their respective dose constraints. Significant amount of clinical experience has been accumulated with this approach during the past century, and it remains the essential component of locally advanced cervix cancer treatment in the majority of centers worldwide [1,2]. While local control rates of conventional BT for small tumors are encouraging, ranging up to $80-95 \%$, the results for the locally advanced disease remain suboptimal [3]. Efforts to improve results of conventional BT in these patients by local dose escalation and perineal interstitial techniques were impeded by a considerable risk of serious late complications [4-7]. Sectional imaging has been implemented into BT planning during past decade at a growing number of centers. 3D image guided adaptive brachytherapy (IGABT) enables a comprehensive assessment of the tumor size and topography at diagnosis and at each BT fraction. It allows for an assessment of correlations between the dose-volume parameters and effects for the target volume and the organs at risk (OAR). This is a more valid concept than the conventional assessment of correlations between point-doses and tissue effects. Treatment plan optimization in the context of IGABT refers to the individual tailoring of irradiation, applying high doses to the target volume while respecting the OAR dose constraints [8,9]. The dosimetric benefits of this approach have been shown to translate into improved rates of uncomplicated cure when compared to the conventional BT $[10,11]$. Currently, magnetic resonance imaging (MRI) represents the modality of choice for IGABT. Accurate interpretation of pre-treatment MRI findings is a precondition for proper understanding of the clinical and radiological disease extent and topography at time of IGABT. Planning of the application technique and optimal timing of the procedure begins already before treatment and may need to be adapted during external beam irradiation (EBRT) according to the clinical and eventual addition- 
al radiological findings. The level of MRI utilization in IGABT differs between individual centers, ranging from no use at all to repetitive imaging during EBRT and at each IGABT fraction. These differences reflect the various infrastructural capabilities, institutional policies, and economic constraints of individual centers. This article summarizes the role of different imaging modalities in cervix cancer IGABT, and outlines the practical aspects of MRI interpretation of the primary tumor extent and topography at BT from the radiation oncologist's perspective. The interpretation of the imaging pathology of the regional nodes is not addressed here.

\section{Imaging modalities}

The adaptive concept of cervix cancer IGABT is based on repetitive clinical and imaging interpretation of tumor extent and topography at diagnosis, its regression during chemo-radiotherapy, and the residual pathological tissues at each BT fraction. In IGABT, the dose is adapted to a target which changes in size and shape from application to application, and the ability for an accurate delineation of the regions of interest is a precondition for treatment success and consistent reporting. Various imaging modalities have been employed in gynecological IGABT [8,9, 12-23].

The role of ultrasound (US) is currently limited mainly to the real-time or off-line guidance of the applicator insertion. Optimal positioning of the BT catheters in the target volume is a pre-requisite for achieving an optimal dose distribution during treatment planning. US-guided placement of the intracavitary and interstitital applicators is an effective and inexpensive method to prevent organ perforation [12-15,24], and to achieve an optimal implant geometry $[16,17]$. While US can add important information to clinical findings with regard to the definition of the extent and topography of the target volume and organs at risk at BT, its utility in treatment planning currently remains limited. Further development of the US-based contouring concepts, sonographic devices, ultrasound probes, applicators, and their set-up and compatibility are required to meet the specific demands of ultrasound imaging with the applicator in place.

Computed tomography (CT) is a relatively inexpensive and accessible modality for IGABT. However, it is characterized by a low ability to discriminate between soft tissues. It demonstrates limited value in differentiation between the tumor and normal cervix, uterus, parametria and vagina, and delineation of the organ walls [2530]. Target volume contours, as outlined on post-insertion $\mathrm{CT}$, can significantly overestimate the tumor width when compared with MRI [25]. The role of CT as the only imaging modality in IGABT of locally advanced cervix cancer is therefore limited. CT is traditionally considered to be adequate for depiction of the outer organ contours, allowing for a reliable estimation of the dose-volume parameters for the OAR [25]. However, in daily clinical experience, the ability of MRI to differentiate the organ walls from organ contents and its high soft tissue depiction quality result in more accurate delineation of the outer organ contours. In spite of the above listed limitations of $\mathrm{CT}$, replacement of the X-ray based approach with the CT-assisted IGABT accompanied by careful incorporation of clinical findings [26], enables the essential qualitative leap from the 2D to 3-4D treatment planning strategy $[27,28]$. Utilization of a pre-brachytherapy MRI without the applicator in place, followed by a post-insertion CT could be encouraged whenever possible to facilitate the CT-based contouring by incorporation of the MRI-findings on the CT images. In addition, the combination of the MRI at first BT fraction, followed by CT at subsequent fraction(s) has been proposed as a feasible and effective strategy to cost reduction and improved availability of cervix cancer IGABT [29].

Incorporation of 18F-fluorodeoxyglucose positron emission tomography (FDG PET) findings and implementation of new tracers for angiogenesis, hypoxia, etc., represent an exciting area of research to identify target (sub)volumes that may require different dose levels. Functional imaging based IGABT should be currently considered an experimental approach.

Magnetic resonance imaging (MRI) is characterized by superior soft tissue depiction quality when compared with CT, capability of multi-planar imaging and absence of ionizing irradiation. No intravenous contrast is needed for adequate visualization and delineation of the regions of interest at IGABT. The inter-reader agreement and correlation with pathological findings in operable cervix cancer has been shown to be superior for MRI when compared with CT [25-36]. Following the early reports on the use of MRI in BT planning in the 1990s [37-40], we have witnessed a systematic development of the concepts and terms in various domains of the MRI-based IGABT in the past decade. This development has resulted in publication of reports on the optimal imaging sequences and image orientations, and recommendations on the MRI assessment of GTV and CTV, 3D dose volume parameters, radiation physics, radiobiology, applicator reconstruction, and MR imaging protocols at diagnosis and BT $[8,9,41-44]$. Favorable reports on the dosimetric outcome of MRI-assisted IGABT are being reflected in a growing body of evidence, demonstrating encouraging clinical results when compared to the best results of conventional BT $[10,11]$. Recently, uncertainties related to the various steps of the IGABT process have been quantified, facilitating the critical interpretation of the results of clinical studies and treatment reporting [45-49]. In summary, MRI is currently considered the imaging modality of choice for cervix cancer IGABT. Implementation of MRI into the BT treatment planning poses several challenges to the radiation oncology team, including the requirements related to the infrastructure, equipment, imaging protocols, training, quality assurance, and interpretation of imaging findings.

\section{Practical aspects of the interpretation of MRI findings at time of brachytherapy}

Recently published recommendations from the Gynaecological GEC-ESTRO Working Group summarize the expert opinion on the basic principles and parameters for MR imaging in cervix cancer IGABT, and propose dedi- 
cated imaging protocols for both the "pre-treatment examination" and "BT examination" [42]. Implementation of published protocols, while taking the specific institutional practice and experience into account, is needed to meet the specific demands of cervix cancer IGABT. Once the post-insertion pelvic MRI data set is obtained, a systematic approach to the interpretation of images can be recommended to assure rapid identification of potentially hazardous conditions, evaluate the adequacy of the BT application, assess the degree of treatment response, and facilitate accurate contouring.

\section{General principles}

The general principles of post-insertion pelvic MRI interpretation include the need for synchronous viewing of images in multiple planes, availability of the pre-treatment MRI, and full description of clinical findings at diagnosis and BT. The incorporation of clinical judgment when assessing the imaging findings cannot be overemphasized, in particular with regard to interpretation of the anatomical regions which are accessible to inspection, palpation, and endoscopy (vulva, vaginal walls, portio, bladder, and rectum). The imaging pathology at BT should always be explained in the context of findings at diagnosis. Intra-departmental consultation within the designated team of radiation oncologists and cooperation with the diagnostic radiologist and radiophysicist is advised whenever possible to minimize uncertainties through consensus opinions.

\section{Perforation of hollow organs}

Uterine perforation should be excluded immediately following the acquisition of the post-insertion sectional images (Fig. 1A). Although most cases of uterine perfo- ration will resolve spontaneously without consequences after conservative treatment, specific attention is required to avoid subsequent complications such as infection, hemorrhage or peritoneal tumor-cell seeding [24,50,51]. Importantly, in an unrecognized uterine perforation, the applicator may come in close contact with the OAR. If this is not accounted for, excessive irradiation of the OAR and under-dosage of the target volume may occur, resulting in serious acute and chronic complications, and reduced probability of local control.

Reported incidence of the uterine perforations during intracavitary BT ranges from 2-14\% [14,50,52,53]. Several predisposing factors for this complication have been identified in the literature, including necrotic cervical tumor, cervical polyp, submucosal myoma, stenosis/distortion of cervical canal, prior conization, retroflexed or extremely anteflexed uterus and age over 60 years [14,24,50, $52,53]$. In the largest series by Šegedin et al., reporting on 428 gynecological applications, uterine perforation was indentified on post-insertion 3D imaging in 3\% of procedures [24]. Most common perforation site was the posterior uterine wall (70\%). In all cases, at least one of the above listed predisposing factors was present. The most commonly identified risk factors were age over 60 years and necrosis and distortion of cervical canal. All cases of uterine perforation in this series were treated conservatively by removing the applicator and monitoring the patient for 24 hours. Prophylactic antibiotics were administered in $62 \%$ of patients and blood transfusion required in $20 \%$. All patients completed their radiotherapy as planned, without further complications. Re-perforation was avoided by using the information from 3D imaging and with real-time guidance of insertion with abdominal and/or transrectal US. At the last follow up, there were no signs of tumor-cell seeding in the affected patients
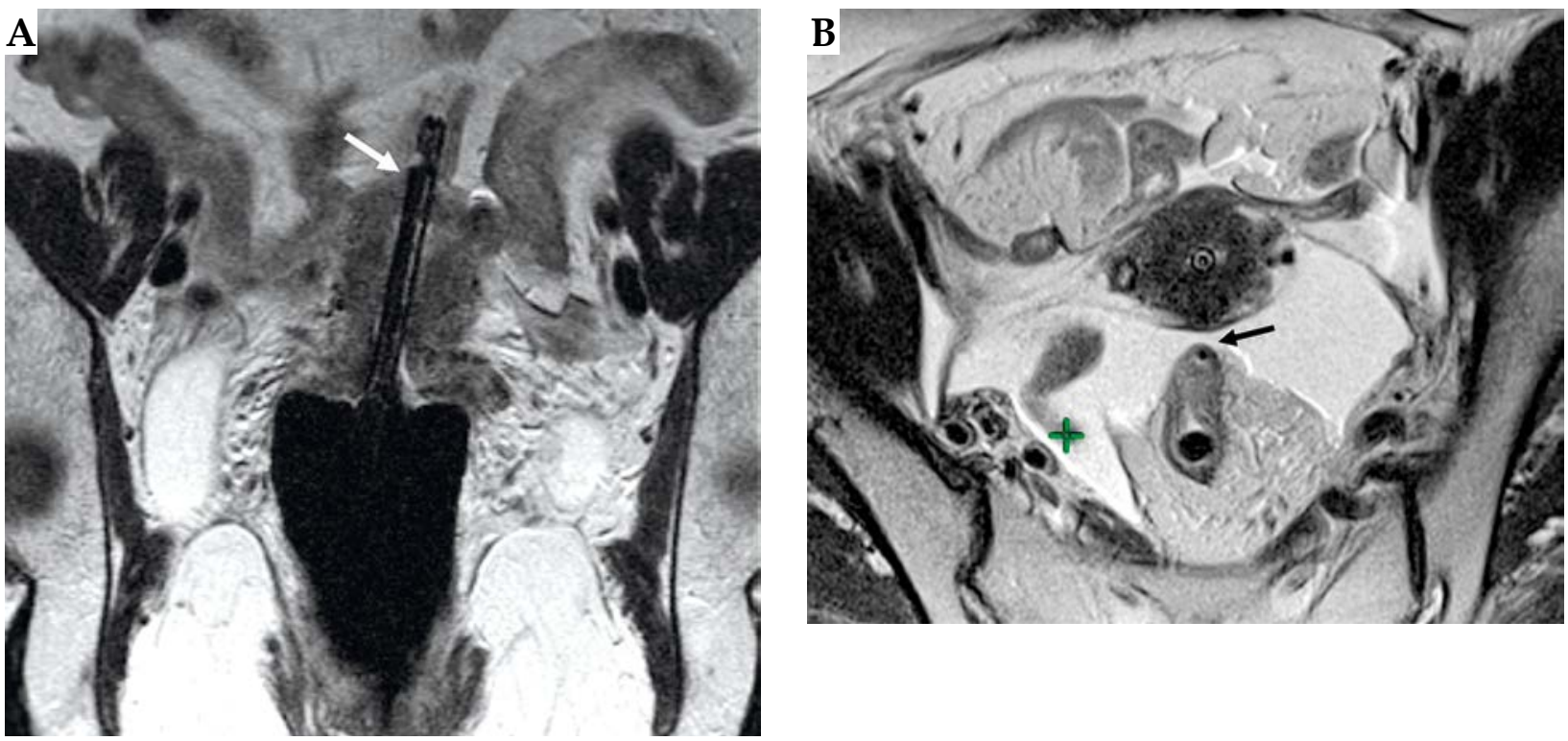

Fig. 1. Two examples of the application-induced organ perforation, identified on post-insertion pelvic T2 weighted FSE MRI. A) Para-coronal (parallel to the uterine tandem) image orientation, revealing perforation of the uterine fundus with the tandem applicator (white arrow). B) Para-transverse (perpendicular to the uterine tandem) image orientation, demonstrating perforation of the rectal wall with a plastic interstitial needle (black arrow) 
[24]. It has to be pointed out that in the series of Šegedin et al., pulsed dose rate BT with long overall treatment times was used [24]. In selected cases, treated with high dose rate technique, BT could potentially be completed before the applicator removal if the patient's general medical condition allows and the planning aims are met during dose optimization.

With increasing use of combined intracavitary and interstitial application techniques, there is an increasing risk of perforation or injury of pelvic hollow organs and blood vessels with the needle applicators (Fig. 1B). There are scarce reports in the literature on the incidence and clinical significance of such complications. Immediate appropriate action would depend on the specific clinical situation and consultation with specialist. It has to be emphasized that specific adaptation of the interstitial application technique is recommended to minimize the chance of perforation of pelvic hollow organs or vessels [54,55]. The use of sharp interstitial needles should be avoided. Needles with blunt tips have been developed and made available specifically for gynecological IGABT. Their insertion has been proven safe, feasible, accurate, and reproducible $[54,55]$.

\section{Adequacy of the implant}

Accurate insertion of the intracavitary applicator and optimal geometric distribution of eventual interstitial needles is a prerequisite for tight control of the dose distri- bution during treatment planning. The ability to achieve the dosimetric planning aim for the target volume while respecting the OAR dose constraints depends on the geometric adequacy of the implanted applicator channels. The inadequate dosimetric consequences of a suboptimal application cannot always be compensated by treatment plan optimization. In practice, the decision on the application technique and geometry of the inserted channels is typically based on clinical and MRI findings at diagnosis and clinical findings at IGABT. MRI for treatment planning is performed only after the application, limiting the ability for corrections in case of suboptimal implant geometry. To overcome these limitations and to increase the likelihood of optimal implant geometry already at the first application, some authors proposed an MRI-assisted pre-planning strategy [56,57]. However, due to the low availability of the MRI, relative complexity of pre-planning and scarcity of published data to support its routine clinical use, this strategy remains limited to certain specialized institutions or clinical studies. In daily clinical practice, we recommend rapid identification of the eventual inadequacies of the implant geometry on post-insertion MRI immediately following their acquisition. Images should be interpreted jointly by the radiation oncologist and radiophysicist, taking into account the spatial inter-relations between the applicator, target volume, and OAR. Such joint assessment by an experienced team will enable identification of the implant deficiencies based on the predicted isodose distribution, even before the actual treatment planning. If a suboptimal
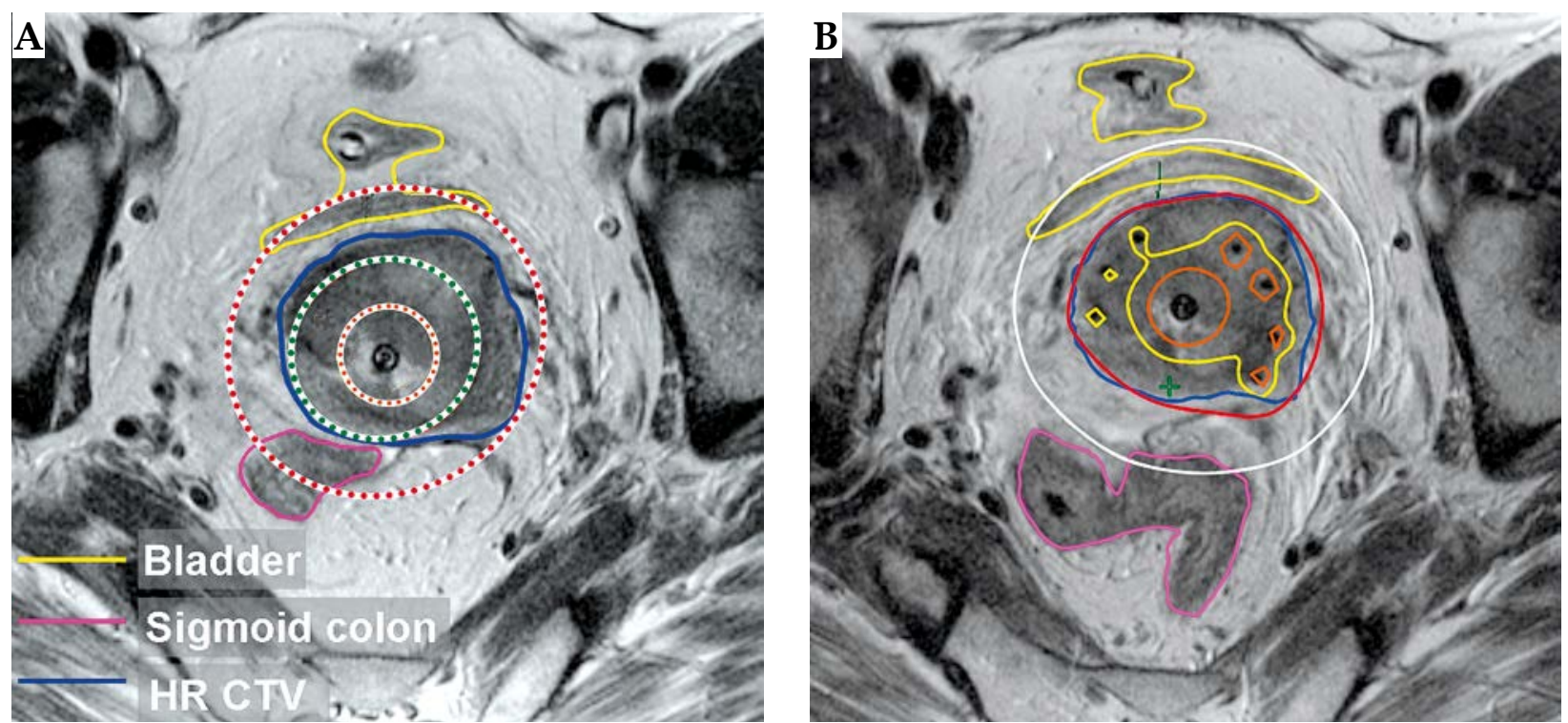

Fig. 2. Assessment of the geometric adequacy of an implant. T2 weighted FSE pelvic MRI with the applicator in place in paratransverse (perpendicular to the uterine tandem) orientation. A) Inadequate implant geometry. Assessment of the post-insertion images reveals a large and topographically unfavourable residual tumor. The isodose distribution resulting from the loading of the intracavitary applicator can be predicted without the actual use of the treatment planning system. Dotted lines represent various theoretical positions of the prescribed isodose. Due to the limited degrees of freedom afforded by the intracavitary applicator, complete HR-CTV coverage with the prescribed dose cannot be achieved without exceeding the normal tissue dose constraints (red dotted line). Similarly, sparing of the OAR can only be accomplished if the HR-CTV is underdosed (green dotted line). B) Adequate implant geometry. Taking the inadequacies of the first application into account, interstitial needles were inserted into the target volume in addition to the intracavitary applicator at the subsequent BT fraction. Treatment plan optimization resulted in a near complete coverage of the HR-CTV with the prescribed dose (red line) while respecting the dose constraints of the OAR 
implant geometry is identified (Fig. 2A), the decision will depend on the specific clinical situation; in principle, one of the following scenarios could be proposed: (1) treatment is completed in spite of suboptimal dose distribution, possibly with a lower dose, followed by an optimized implant and application of higher dose during next BT fraction, improving the cumulative dosimetric outcome; (2) the patient is brought back to the operating room and the implant geometry is optimized, followed by application of the planned treatment; (3) the applicator is removed and the geometrically adequate implant and treatment is carried out during subsequent insertion(s) (Fig. 2B).
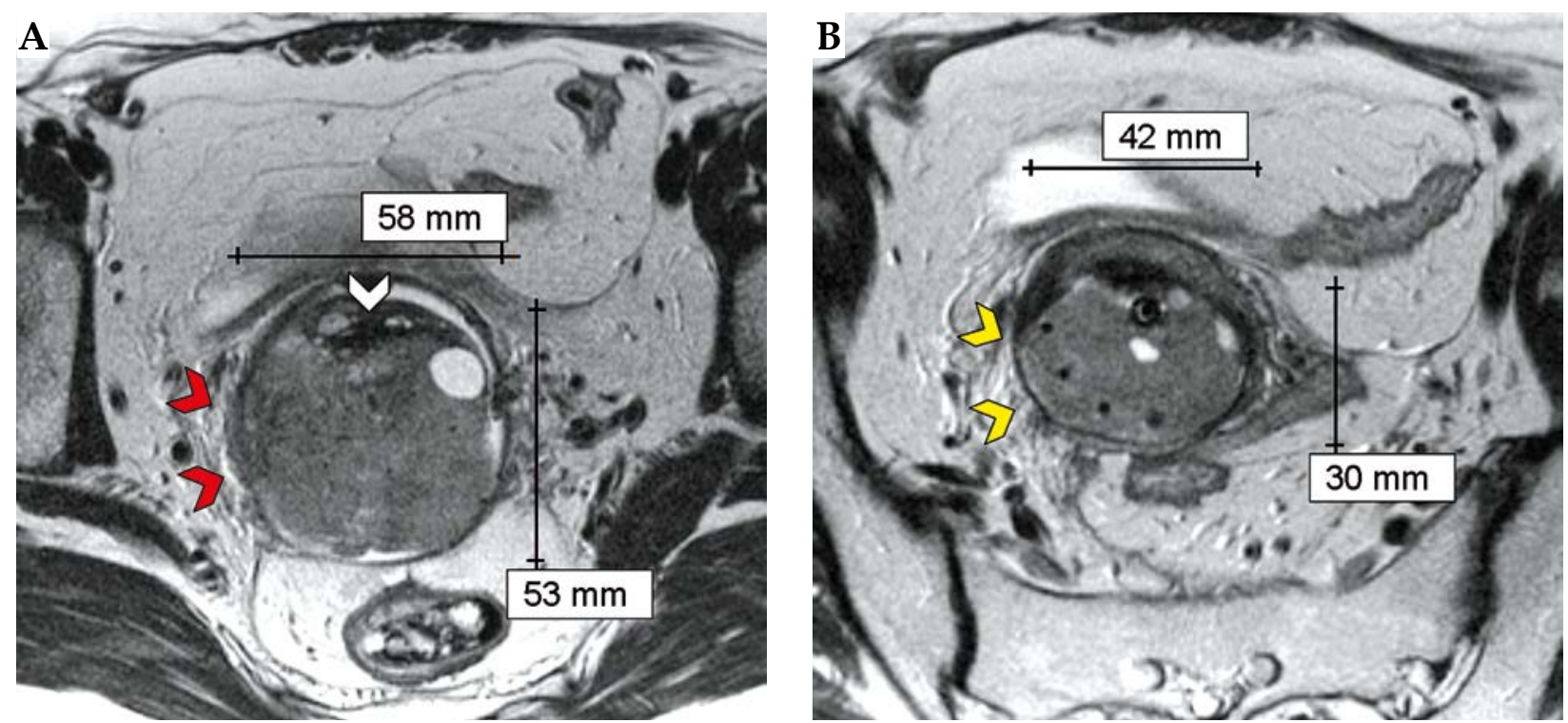

Fig. 3. Para-transverse (perpendicular to the cervical canal) T2 weighted FSE MRI of a stage IIB cervix cancer. A) MRI at diagnosis. A large lesion of high signal intensity is identified in the cervix. The pattern of tumor growth is predominantly expansive. There are minimal signs of parametrial infiltration on the right side, indicated by the disappearance of the cervical stromal rim, and spiculary tumor projections into the parametrium (red arrows). The epicenter of the tumor is located posteriorly with a small region of normal appearing stroma in the anterior aspect of the cervix (white arrow). B) At time of brachytherapy, reconstitution of the low signal intensity cervical stroma can be appreciated anteriorly and surrounding the residual gross tumor as a thin rim at its posterior and lateral aspects. There are no residual pathological findings in the parametria (grey zones). The topography at BT corresponds to the initial pattern of growth with the residual tumor located posteriorly. Adequate implant geometry
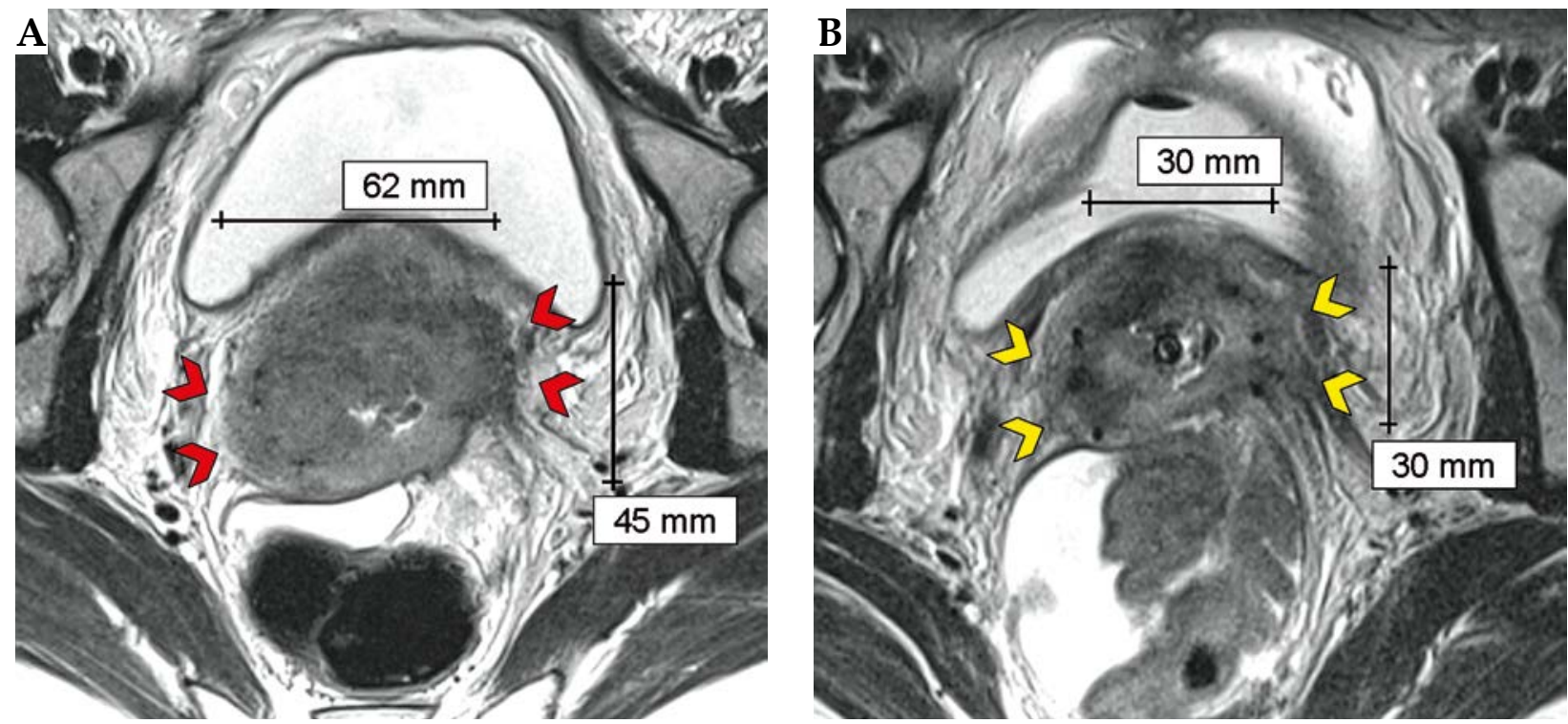

Fig. 4. Para-transverse (perpendicular to the cervical canal) T2 weighted FSE MRI of a stage IIIB cervix cancer. A) MRI at diagnosis. The pattern of tumor growth is predominantly infiltrative with bilateral gross parametrial invasion (red arrows). B) At time of brachytherapy, bilateral residual pathological tissues in the parametria (grey zones) are identified, corresponding to the areas of initial gross tumor invasion. There is patchy reconstitution of the low signal of the cervical stroma, located mainly anteriorly and on the right. Adequate implant geometry 


\section{Tumor response and target volume assessment}

Accurate and meaningful interpretation of the imaging findings at BT is a precondition for appropriate selection of the tissues to be included in the target volumes during the delineation process. The GEC-ESTRO target concept at time of BT includes the gross tumor volume (GTV), high-risk clinical target volume (HR-CTV), and intermediate risk clinical target volume (IR-CTV). The target volumes are defined at each BT, according to the changes of tumor size and topography during time and reflecting the adaptive treatment concept $[8,58]$. Systematic approach to interpretation of imaging and clinical findings is recommended at time of BT in order to minimize the contouring uncertainties and errors. This process should begin already before the actual delineation, and includes careful interpretation of clinical and imaging findings at BT in the context of initial findings. The extent and topography of residual GTV, eventual areas of necrosis, and residual pathological tissues in the parametria, vagina and uterus (the "grey zones") should be systematically evaluated and treatment response quantified. During this evaluation, it should be taken into account that it is not expected to find tumor or residual pathological tissues in the regions that were tumor-free at initial examination (Figs. 3 and 4). In a study by Schmid et al., residual pathological MRI findings in the parametria were identified in $19 \%$ of the cases with predominantly expansive initial tumor growth and in $68-90 \%$ of initially infiltrative tumors, depending on the degree of infiltration [59]. Therefore, initial MRI characteristics of the parametrial infiltration by the cervical tumor appear to allow prediction of the tumor response during external beam radiotherapy and chemotherapy.

\section{Conclusions}

Magnetic resonance imaging is the gold-standard imaging modality for cervix cancer IGABT. Radiation oncologist's perspective of MRI interpretation at time of BT is specific and differs from the radiologist's perspective. It is characterized by the need for a detailed definition of the border between the target volume and the surrounding normal tissues during contouring, since the dose that is delivered to the tissues following treatment plan optimization directly depends on the delineated regions of interest. Intra-departmental consultation within the team of radiation oncologists and cooperation with the diagnostic radiologist and radiophysicist is advised to minimize uncertainties through consensus opinions. Systematic evaluation of the post-insertion MRI is recommended and should begin with immediate identification and treatment of eventual complications of the application procedure, such as perforation of hollow organs and/or vessels. Next, the adequacy of the implant should be rapidly assessed to guide decisions about eventual corrective measures. Finally, systematic evaluation of the tumor extent, topography, and treatment response is performed as a basis for an appropriate selection and delineation of the target volumes. Accurate and reproducible delineation of the GTV and CTV is required to take full advantage of this high precision treatment technique, minimize uncertainties and assure consistent recording and reporting of treatment.

\section{Disclosure}

Authors report no conflict of interest.

\section{References}

1. Guedea F, Venselaar J, Hoskin P et al. Patterns of care for brachytherapy in Europe: updated results. Radiother Oncol 2010; 97: 514-520.

2. Viswanathan AN, Erickson BA. Three-dimensional imaging in gynaecological brachytherapy: a survey of the American Brachytherapy Society. Int J Radiat Oncol Biol Phys 2010; 76: 104-109.

3. Gerbaulet A, Pötter R, Haie-Meder C. Cervix cancer. In: Gerbaulet A, Pötter R, Mazeron JJ, Meertens H, Van Limbergen E (eds.). The GEC ESTRO Handbook of Brachytherapy. European Society of Therapeutic Radiology and Oncology, Brussels 2002; 300-363.

4. Martinez A, Edmundson GK, Cox RS et al. Combination of external beam irradiation and multiple-site perineal applicator (MUPIT) for treatment of locally advanced or recurrent prostatic, anorectal, and gynaecologic malignancies. Int J Radiat Oncol Biol Phys 1985; 11: 391-398.

5. Hughes-Davies L, Silver B, Kapp DS. Parametrial interstitial brachytherapy for advanced or recurrent pelvic malignancy: The Harvard/Stanford experience. Gynecol Oncol 1995; 58: 24-27.

6. Aristizibal SA, Surwit EA, Hevezi JM et al. Treatment of advanced cancer of the cervix with transperineal interstitial irradiation. Int J Radiat Oncol Biol Phys 1983; 9: 1013-1107.

7. Monk BJ, Tewariu K, Burger RA et al. A comparison of intracavitary versus interstitial irradiation in the treatment of cervical cancer. Gynacol Oncol 1997; 67: 241-247.

8. Haie-Meder C, Pötter R, Van Limbergen E et al. Recommendations from Gynaecological (GYN) GEC-ESTRO Working Group (I): concepts and terms in 3D image based 3D treatment planning in cervix cancer brachytherapy with emphasis on MRI assessment of GTV and CTV. Radiother Oncol 2005; 74: 235-245.

9. Pötter R, Haie-Mader C, Van Limbergen E et al. Recommendations from gynaecological (GYN) GEC-ESTRO Working Group: (II): concepts and terms of 3D imaging, radiation physics, radiobiology, and $3 \mathrm{D}$ dose volume parameters. $\mathrm{R} a$ diother Oncol 2006; 78: 67-77.

10. Lindegaard JC, Tanderup K, Nielsen SK et al. MRI-Guided 3D Optimization Significantly Improves DVH Parameters of Pulsed-Dose-Rate Brachytherapy in Locally Advanced Cervical Cancer. Int J Radiat Oncol Biol Phys 2008; 71: 756-764.

11. Pötter R, Georg P, Dimopoulos JCA et al. Clinical outcome of protocol based image (MRI) guided adaptive brachytherapy combined with 3D conformal radiotherapy with or without chemotherapy in patients with locally advanced cervical cancer. Radiother Oncol 2011; 100: 116-123.

12. Mayr NA, Montebello JF, Sorosky JI et al. Brachytherapy management of the retroverted uterus using ultrasound-guided implant applicator placement. Brachytherapy 2005; 4: 24-29.

13. Sahinler I, Cepni I, Colpan D et al. Tandem application with transvaginal ultrasound guidance. Int J Radiat Oncol Biol Phys 2004; 59: 190-196.

14. Granai CO, Allee P, Doherty F et al. Ultrasound used for assessing the in situ position of intrauterine tandems. Gynecol Oncol 1984; 18: 334-338.

15. Davidson MT, Yuen J, D'Souza D et al. Optimization of highdose-rate cervix brachytherapy applicator placement: the 
benefits of intraoperative ultrasound guidance. Brachytherapy 2008; 7: 248-253.

16. Stock RG, Chan K, Terk M et al. A new technique for performing Syed-Neblett template interstitial implants for gynecologic malignancies using transrectal-ultrasound guidance. Int J Radiat Oncol Biol Phys 1997; 37: 819-825.

17. Weitmann HD, Knocke TH, Waldhäusl C et al. Ultrasound-guided interstitial Brachytherapy in the treatment of advanced vaginal recurrences from cervical and endometrial carcinoma. Strahlentherapie und Onkologie 2006; 182: 86-95.

18. Van Dyk S, Narayan K, Fisher R et al. Conformal brachytherapy planning for cervical cancer using transabdominal ultrasound. Int I Radiat Oncol Biol Phys 2009; 75: 64-70.

19. Datta NR, Srivastava A, Maria Das KJ et al. Comparative assessment of doses to tumor, rectum, and bladder as evaluated by orthogonal radiographs vs. computer enhanced computed tomography-based intracavitary brachytherapy in cervical cancer. Brachytherapy 2006; 5: 223-229.

20. Shin KH, Kim TH, Cho JK et al. CT-guided intracavitary radiotherapy for cervical cancer: Comparison of conventional point A plan with clinical target volume-based three-dimensional plan using dose volume parameters. Int J Radiat Oncol Biol Phys 2006; 64: 197-204.

21. Mai J, Rownd J, Erickson B. CT-guided high-dose-rate dose prescription for cervical carcinoma: the importance of uterine wall thickness. Brachytherapy 2002; 1: 27-35.

22. Pelloski CE, Palmer M, Chronowski GM et al. Comparison between CT-based volumetric calculations and ICRU reference-point estimates of radiation doses delivered to bladder and rectum during intracavitary radiotherapy for cervical cancer. Int J Radiat Oncol Biol Phys 2005; 62: 131-137.

23. Sun LM, Huang EY, Ko SF et al. Computer-tomography-assisted three-dimensional technique to assess rectal and bladder wall dose in intracavitary brachytherapy for uterine cervical cancer. Radiother Oncol 2004; 71: 333-337.

24. Šegedin B, Gugić J, Petrič P. Uterine perforation - 5-year experience on 3D image guided gynaecological brachytherapy at Institute of Oncology Ljubljana. Radiol Oncol 2013; 47: 154-160.

25. Viswanathan AN, Dimopoulos JCA, Kirisits C et al. CT versus MRI-based contouring in cervical cancer brachytherapy: results of a prospective trial and preliminary guidelines for standardized Contours. Int J Radiat Oncol Biol Phys 2007; 68: 491-498.

26. Hegazy N, Pötter R, Kirisits C et al. High-risk clinical target volume delineation in CT-guided cervical cancer brachytherapy: impact of information from FIGO stage with or without systematic inclusion of 3D documentation of clinical gynecological examination. Acta Oncol 2013; 52: 1345-1352.

27. Tyagi K, Mukundan H, Mukherjee D et al. Non isocentric film-based intracavitary brachytherapy planning in cervical cancer: a retrospective dosimetric analysis with CT planning. J Contemp Brachyther 2012; 3: 129-134.

28. Anderson J, Huang Y, Kim Y. Dosimetric impact of point A definition on high-dose-rate brachytherapy for cervical cancer: evaluations on conventional point A and MRI-guided, conformal plans. J Contemp Brachyther 2012; 4: 241-246.

29. Nesvacil N, Pötter R, Sturdza A et al. Adaptive image guided brachytherapy for cervical cancer: a combined MRI-CTplanning technique with MRI only at first fraction. Radiother Oncol 2013; 107: 75-81.

30. Mitchell DG, Snyder B, Coakley F et al. Early invasive cervical cancer: tumor delineation by magnetic resonance imaging, computed tomography, and clinical examination, verified by pathologic results, in the ACRIN 6651/GOG 183 Intergroup Study. J Clin Oncol 2006; 24: 5687-5694.

31. Oszarlak O, Tjalma W, Scheppens E et al. The correlation of preoperative CT, MR imaging, and clinical staging (FIGO) with histopathology findings in primary cervical carcinoma. Eur Radiol 2003; 13: 2338-2345.

32. Jung DC, Ju W, Choi $\mathrm{HJ}$ et al. The validity of tumour diameter assessed by magnetic resonance imaging and gross specimen with regard to tumour volume in cervical cancer patients. Eur J Cancer 2008; 44: 1524-1528.

33. Bipat S, Glas AS, van der Velden J et al. Computed tomography and magnetic resonance imaging in staging of uterine cervical carcinoma: a systematic review. Gynecol Oncol 2003; 91: 59-66.

34. Hricak H, Gatsonis C, Coakley FV et al. Early invasive cervical cancer: CT and MR imaging in preoperative evaluation ACRIN/GOG comparative study of diagnostic performance and interobserver variability. Radiology 2007; 245: 491-498.

35. Dimopoulos JCA, Schard G, Berger D et al. Systematic Evaluation of MRI Findings in different Stages of Treatment of Cervical Cancer: Potential of MRI on Delineation of Target, Patho-Anatomical Structures and Organs at Risk. Int J Radiat Oncol Biol Phys 2006; 64: 1380-1388.

36. Dimopoulos JCA, Schirl G, Baldinger A et al. MRI assessment of cervical cancer for adaptive radiotherapy. Strahlenther Onkol 2009; 185: 282-287.

37. Schoeppel SL, LaVigne ML, Martel MK et al. Three-dimensional treatment planning of intracavitary gynaecologic implants: analysis of ten cases and implications for dose specification. Int J Radiat Oncol Biol Phys 1994; 28: 277-283.

38. Schoeppel SL, Ellis JH, LaVigne ML et al. Magnetic resonance imaging during intracavitary gynecologic brachytherapy. Int J Radiat Oncol Biol Phys 1992; 23: 169-174.

39. Schmidt BF, Hirnle P, Kaulich TW et al. The value of NMR tomography in the planning of HDR-afterloading brachytherapy in cervical carcinomas. The experience with 41 patients. Rofo 1991; 155: 109-116.

40. Tardivon AA, Kinkel K, Lartigau E et al. MR imaging during intracavitary brachytherapy of vaginal and cervical cancer: preliminary results. Radiographics 1996; 16: 1363-1370.

41. Hellebust TP, Kirisits C, Berger D et al. Recommendations from Gynaecological (GYN) GEC-ESTRO Working Group: Considerations and pitfalls in commissioning and applicator reconstruction in 3D image-based treatment planning of cervix cancer brachytherapy. Radiother Oncol 2010; 96: 153-160.

42. Dimopoulos JC, Petrow P, Tanderup K et al. Recommendations from Gynaecological (GYN) GEC-ESTRO Working Group (IV): Basic principles and parameters for MRI imaging within the frame of image based adaptive cervix cancer brachytherapy. Radiother Oncol 2012; 103: 113-122.

43. Dempsey C, Arm J, Best L et al. Optimal single 3T MR imaging sequence for HDR brachytherapy of cervical cancer. J Contemp Brachytherapy 2014; 6: 3-9.

44. Petrič P, Dimopoulos JCA, Kirisits C et al. Inter- and intraobserver variation in HR-CTV contouring: intercomparison of transverse and paratransverse image orientation in 3D-MRI assisted cervix cancer brachytherapy. Radiother Oncol 2008; 89: 164-171.

45. Petrič P, Hudej R, Rogelj P et al. Uncertainties of target volume delineation in MRI guided adaptive brachytherapy of cervix cancer: A multi-institutional study. Radiother Oncol 2013; 107: 6-12

46. Hellebust TP, Tanderup K, Lervåg C et al. Dosimetric impact of interobserver variability in MRI-based delineation for cervical cancer brachytherapy. Radiother Oncol 2013; 107: 13-19.

47. Tanderup K, Nesvacil N, Pötter R et al. Uncertainties in image guided adaptive cerix cancer brachytherapy: Impact on planning and prescription. Radiother Oncol 2013; 107: 1-5.

48. Nesvacil N, Tanderup K, Hellebust TP et al. A multicentre comparison of the dosimetric impact of inter- and intra-frac- 
tional anatomical variations in fractionated cervix cancer brachytherapy. Radiother Oncol 2013; 107: 20-25.

49. Lang S, Nesvacil N, Kirisits C et al. Uncertainty analysis for 3D image-based cervix cancer brachytherapy by repetitive MR imaging: Assessment of DVH-variations between two HDR fractions within one applicator insertion and their clinical relevance. Radiother Oncol 2013; 107: 26-31.

50. Kim RY, Levy DS, Brascho DJ et al. Uterine perforation during intracavitary application. Prognostic significance in carcinoma of the cervix. Radiology 1983; 147: 249-251.

51. Mills SE, Sugg NK, Mahnesmith RC. Endometrial carcinoma with pelvic involvement following uterine perforation. Diagn Gynecol Obstet 1981; 3: 149-154.

52. Matsuyama T, Tsukamoto N, Matsukuma K, Kamura T, Jingu K. Uterine perforation at the time of brachytherapy for the carcinoma of the uterine cervix. Gynecol Oncol 1986; 23 : 205-211.

53. Barnes EA, Thomas G, Ackerman I et al. Prospective comparison of clinical and computed tomography assessment in detecting uterine perforation with intracavitary brachytherapy for carcinoma of the cervix. Int J Gynecol Cancer 2007; 17: 821-826.

54. Dimopoulos JCA, Kirisits C, Petric P et al. The Vienna applicator for combined intracavitary and interstitial brachytherapy of cervical cancer: clinical feasibility and preliminary results. Int J Radiat Oncol Biol Phys 2006; 66: 83-90.

55. Kirisits C, Lang S, Dimopoulos J et al. The Vienna applicator for combined intracavitary and interstitial brachytherapy of cervical cancer: design, application, treatment planning and dosimetric results. Int J Radiat Oncol Biol Phys 2006; 65: 624-630.

56. Petric, P, Hudej R, Music M. MRI assisted cervix cancer brachytherapy pre-planning, based on insertion of the applicator in para-cervical anaesthesia: preliminary results of a prospective study. J Contemp Brachyther 2009; 1: 163-169.

57. Fokdal L, Tanderup K, Hokland SB et al. Clinical feasibility of combined intracavitary/interstitial brachytherapy in locally advanced cervical cancer employing MRI with a tan$\mathrm{dem} /$ ring applicator in situ and virtual preplanning of the interstitial component. Radiother Oncol 2013; 107: 63-68.

58. Petric P, Pötter R, Van Limbergen E et al. Adaptive contouring of the target volume and organs at risk. In: Viswanathan AN (ed.). Gynecologic radiation therapy: novel approaches to image-guidance and management. Springer, Heidelberg 2011; 99-118.

59. Schmid MP, Fidarova E, Pötter R et al. Magnetic resonance imaging for assessment of parametrial tumour spread and regression patterns in adaptive cervix cancer radiotherapy. Acta Oncol 2013; 52: 1384-1390. 